\title{
Avaliação ortopédica e ultrassonográfica da estabilidade dos quadris de recém-nascidos encaminhados por pediatras, com suspeita de Displasia Típica do Desenvolvimento.
}

\section{Orthopedic and ultrasound assessment of hip stability of newborns referred by pediatricians with suspected Developmental Dysplasia.}

Mário Augusto Ferreira Cruz ${ }^{1}$ iD; José Batista Volpon ${ }^{1}$ (D)

R E S U M O

\begin{abstract}
Objetivo: avaliar recém-nascidos com suspeita de instabilidade do quadril, encaminhados por pediatras a um serviço ortopédico terciário. Métodos: recém-nascidos de uma maternidade pública universitária, com suspeita de instabilidade ou fatores de risco para displasia do quadril, eram encaminhados ao Departamento de Ortopedia e Anestesiologia, Ribeirão Preto/SP, onde eram avaliados clinicamente e através de exames ultrassonográficos dos quadris. Constatada a displasia, iniciava-se o tratamento, e em casos em que havia apenas imaturidade do quadril e exame clínico normal, procedia-se à observação e re-exame clinico e ultrassonográfico com dois ou três meses de vida. Resultados: foram examinados 448 recém-nascidos, com predominância feminina e média de idade na primeira avaliação de 27 dias. A principal causa do encaminhamento foi apresentação pélvica. Em $8 \%$ havia sinal de Ortolani positivo e em 12,5\% estalido no quadril. No exame ortopédico, 405 (90,5\%) pacientes eram normais, 8,5\% apresentavam estalido no quadril e 1,1\% apresentavam teste de Ortolani positivo. À ultrassonografia, 368 (89,5\%) apresentavam imaturidade, 26 (6,3\%) tinham displasia moderada e em $17(4,1 \%)$ pacientes os quadris eram francamente displásicos. Todos os casos com sinal de Ortolani positivo apresentavam quadro ultrassonográfico de displasia. Conclusão: houve excesso de diagnóstico de instabilidade do quadril na avaliação do pediatra, o que, no entanto, permitiu ao paciente uma segunda avaliação, em ambiente mais especializado e com mais recursos tecnológicos.
\end{abstract}

Descritores: Recém-Nascido. Quadril. Luxação Congênita de Quadril. Luxações Articulares. Instabilidade Articular.

\section{INTRODUÇÃO}

A Displasia do Desenvolvimento do Quadril (DDQ) envolve um espectro de afecções da criança, de caráter evolutivo, que se inicia por instabilidade e displasia acetabular, e pode chegar à subluxação e até mesmo à luxação completa do quadril durante o crescimento. Apresenta uma forma típica, em que a criança é normal sob outros aspectos, e uma forma teratogênica, em que o quadril geralmente está luxado ao nascimento em virtude de quadros sistêmicos sindrômicos ou afecções neuromusculares, como mielomengingocele, artrogripose e outras ${ }^{1}$.

$$
\text { A displasia típica pode regredir }
$$
espontaneamente nos casos leves, mas sem tratamento, pode resultar em quadro doloroso associado à degeneração articular e distúrbios da marcha'.
A incidência de DDQ típica depende da região geográfica e da literatura analisada, com variação de 1/1.000 a 20/1.000 nascidos vivos ${ }^{2-5}$. Em nosso meio, apesar de não existirem estatísticas oficiais, apresenta maior incidência na região sul do país.

No passado, a afecção era conhecida como luxação congênita do quadril, porque o diagnóstico era feito tardiamente e, consequentemente, o tratamento iniciado quando o quadril já estava deslocado, apresentando maus resultados e muitas sequelas. A partir do início do Século $X X$, grande contribuição foi feita por Ortolani, ao divulgar a possibilidade do diagnóstico e tratamento precoces da condição. Ao envolver no diagnóstico médicos não ortopedistas, especialmente os pediatras, Ortolani muito contribuiu para a prevenção de sequelas, até então, muito frequentes no meio ortopédico.

1 - Universidade de São Paulo, Faculdade de Medicina de Ribeirão Preto, Departamento de Ortopedia e Anestesiologia, Ribeirão Preto, SP, Brasil. 
Outro passo histórico importante ocorreu quando foi reconhecida a possibilidade da existência de casos de displasias típicas com diferentes gravidades, bem como o aspecto evolutivo da condição e a possibilidade de nem sempre ser congênita, o que levou à mudança do nome de "luxação congênita do quadril" para "displasia do desenvolvimento do quadril"6,7. Atualmente, o termo "luxação congênita" ficou reservado para os casos de diagnóstico tardio ou aqueles teratogênicos, em que a luxação acontece ainda intraútero e, geralmente, acompanhada de más formações graves dos vários elementos anatômicos do quadril, com deslocamento completo e irredutibilidade por meios conservadores.

Outro grande subsídio para o entendimento da DDQ surgiu com o uso da ultrassonografia, em que se destaca a contribuição de Graf8-11, tornando possível estabelecer os diferentes graus da afecção e selecionar o tratamento. Atualmente, está bem estabelecido que o pediatra é um profissional importante no diagnóstico da DDQ, pois a ele cabe a triagem do recém-nascido com suspeita da afecção, para o encaminhamento ao ortopedista. Em nossa Instituição é longa a parceria entre o pediatra e o ortopedista no sentido de estabelecer o diagnóstico e o tratamento precoces dos casos de DDQ, sendo que os testes de Ortolani e Barlow ${ }^{12,13}$, bem como a presença de sinais de risco, constituem rotina na semiologia do neonato.

Assim, é objetivo da presente investigação avaliar a população de recém-nascidos em uma maternidade pública encaminhada ao ortopedista pediátrico por suspeita de DDQ ou pela presença de fatores de risco. A hipótese é que ocorrem muitos casos suspeitos, porém, sem comprovação diagnóstica com os exames objetivos.

\section{MÉTODOS}

Trata-se de estudo transversal, descritivo, retrospectivo, com abordagem qualitativa e quantitativa do quadril de recém-nascidos (RN) encaminhados de uma maternidade pública de hospital escola. Quando o pediatra suspeitava ou diagnosticava alguma afecção do aparelho locomotor, o RN era enviado para o setor de ortopedia pediátrica da mesma instituição para avaliação ortopédica completa. Na presença de sinais de Ortolani ou Barlow positivos, a criança era imediatamente encaminhada. Entretanto, se não houvesse instabilidade, mas sim presença de sinais de risco para DDQ, como ocorrência familiar, apresentação pélvica, sinais inespecíficos, como estalidos, ou houvesse dúvida quanto à estabilidade do quadril, a criança era encaminhada com quatro semanas de vida para exame do especialista. Este atraso era proposital para permitir que eventuais casos de imaturidade do quadril, e que pudessem simular displasia, tivessem regredido por ocasião do exame ortopédico.

O exame ortopédico especializado era realizado por residentes de Ortopedia do terceiro ano, supervisionados por um ortopedista pediátrico do grupo. Além da avaliação ortopédica geral, o quadril era examinado quanto à assimetria pélvica, presença de pregas glúteas assimétricas (sinal de PeterBade), encurtamento, sinal de Galeazzi, limitação da abdução (Sinal de Hart) e testes de estabilidade (Ortolani/Barlow). Antecedentes familiares positivos para DDQ, apresentação pélvica e gravidez gemelar foram considerados sinais de riscos. Na instabilidade do quadril, era solicitada a ultrassonografia (US) pelo método de Graf, quantificando o grau de displasia, e iniciado o tratamento. Porém, com exame físico normal, mas com presença de fatores de risco, era solicitado o ultrassom dos quadris em torno de um mês de vida. 
As US foram realizadas por médicos residentes radiologistas em especialização em aparelho locomotor, sob supervisão de médico assistente. Foram determinados os ângulos alfa e beta, que, lançados na régua de Graf, delimitam três zonas (Figura 1). Na zona I há apenas imaturidade do quadril e não há necessidade de tratamento específico, mas apenas acompanhamento. A zona Il está subdividida em duas áreas: subzona de transição, em que os pacientes podem ser tratados ou não a depender dos antecedentes e exame físico, e subzona de perigo, em que os casos geralmente são tratados. Os quadris da zona III são considerados amplamente displásicos e requerem tratamento ${ }^{9}$. Neste estudo, foram excluídos casos de luxação teratogênica (associação com síndromes, más formações múltiplas ou doenças neuromusculares).

Para a presente avaliação, os dados foram coletados dos prontuários médicos dos recémnascidos atendidos durante o período compreendido entre junho de 2015 e outubro 2017 (29 meses). A conduta seguiu o fluxograma da figura 2 .

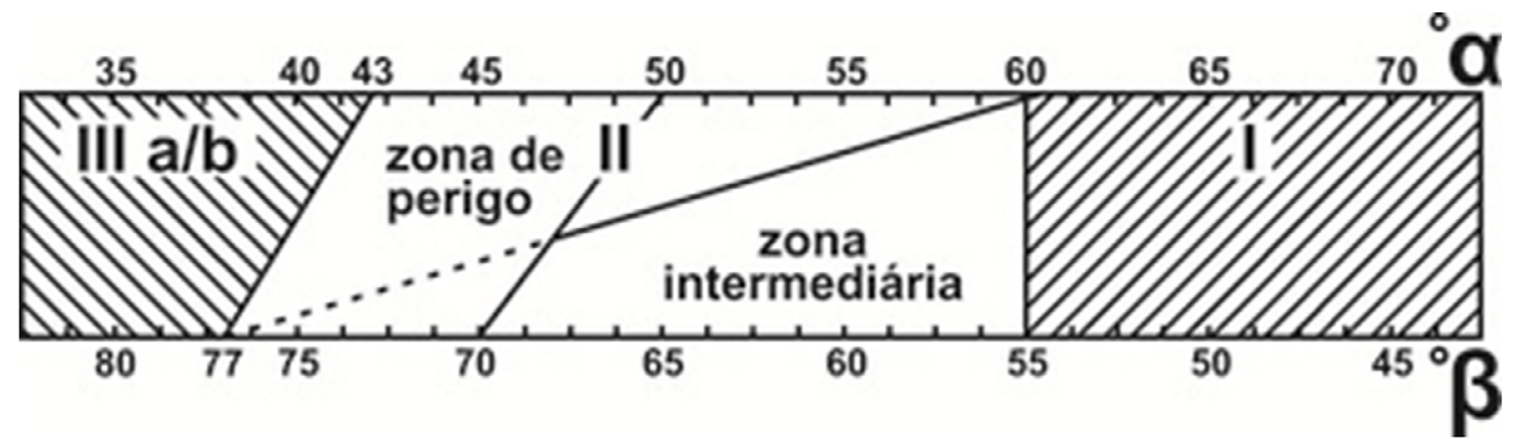

Figura 1. Régua de Graf usada para facilitar a interpretação do grau de displasia e decidir a conduta. No limite superior ficam os valores do ângulo alfa $(\alpha)$ e, no limite inferior os valores de beta (B) (GRAF, 1984). São delimitadas três regiões.

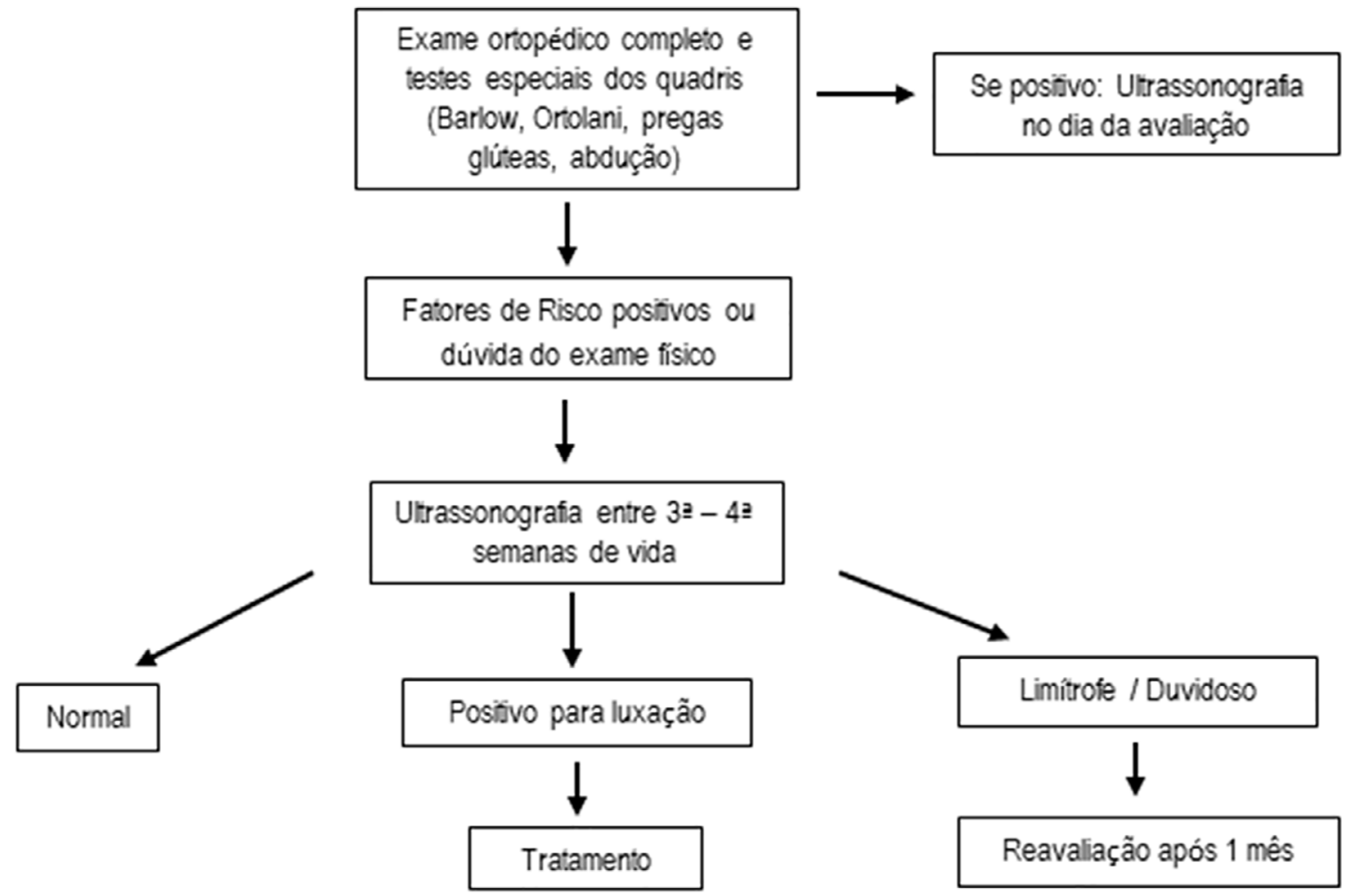

Figura 2. Fluxograma usado para seleção e conduta em todos os casos. 
As variáveis analisadas foram inseridas em uma planilha Excel@ e os dados foram analisados de forma descritiva, por média e desvio padrão (DP).

O presente estudo foi aprovado pelo Comitê de Ética da instituição com número de Certificado de Apresentação para Apreciação Ética (CAAE) 78086117.1.0000.5440.

\section{RESULTADOS}

Foram encaminhados e avaliados pelo ortopedista 448 recém-nascidos com suspeita de alguma alteração no quadril. Destes, 243 (54,3\%) eram do sexo feminino e 205 masculinos. A média de idade na primeira avaliação com o especialista em Ortopedia Pediátrica foi de 27 dias ( $D P \pm 17)$.

O principal motivo de encaminhamento para avaliação foi a apresentação pélvica ao nascimento, correspondendo a 305 recém-nascidos (68\%), dos quais 29 apresentavam alterações menores ao exame físico, ou outros fatores de risco, tais como gravidezes gemelares, história familiar, pé calcâneo valgo ou joelho recurvado. Trinta e seis recém-nascidos (8\%) foram encaminhados com sinal de Ortolani ou Barlow positivos ao nascimento e $56(12,5 \%)$ por apresentarem estalido nos quadris.

A maioria nasceu de parto cesariano $(78,6 \%)$ e a apresentação ao nascimento mais frequente foi a pélvica $(75,2 \%)$, seguida pelas apresentações cefálica $(21,6 \%)$ e transversa $(0,2 \%)$, sendo que em $3 \%$ dos avaliados a informação do tipo de nascimento não foi encontrada. A cor da pele referida ficou distribuída em: 76\% branca, 19\% parda e 5\% negra.

Durante o exame físico ortopédico, em relação ao quadril, os principais achados foram presença de estalido nos quadris em 38 recém-nascidos (8,5\%) e Ortolani/Barlow positivos em cinco (1,1\%). Exame físico normal do quadril foi encontrado em 405 (90,5\%). Dos 448 recém-nascidos encaminhados, 411 foram submetidos ao exame ultrassonográfico dos quadris.
Nos demais, não houve indicação da realização do exame, pois não havia fatores de risco e o exame dos quadris pelo ortopedista foi normal. Desta forma, 37 recém-nascidos não foram submetidos a exames de imagem. A média de idade no momento da realização da US foi de 33 dias $(D P \pm 22,1)$. Dos 411 recém-nascidos avaliados pela US, 368 (89,5\%) apresentavam Graf I (imaturidade), 26 $(6,3 \%)$ apresentavam ao menos um quadril na zona intermediária da régua - Graf II, e 17 (4,1\%) apresentaram sinais de displasia (zona de perigo ou francamente displásicos). Todos os casos com sinal de Ortolani positivo apresentavam quadro ultrassonográfico de displasia. Nesses 17 recémnascidos $(4,1 \%)$ foi estabelecido o diagnóstico de displasia do quadril, sendo todos tratados com os suspensórios de Pavlik ${ }^{14}$ (Figura 3).

\section{DISCUSSÃO}

Embora o conceito da DDQ tenha evoluído, bem como seus métodos de diagnóstico e tratamento, o diagnóstico precoce ainda fica comprometido se não houver conscientização dos médicos pediatras. Esta recomendação é clássica e teve origem com o trabalho de Ortolani, em $1937^{15}$. Previamente, outros pioneiros da Ortopedia, como Pierre Le Damany, trabalhando em maternidades, descreveu, de maneira independente, uma manobra muito semelhante à de Ortolani, a que nomeou "Le signe du ressaut". Este mesmo autor enfatizou a importância do diagnóstico precoce e estabeleceu o princípio da posição de flexão e abdução dos quadris como método de tratamento ${ }^{16}$. Entretanto, o grande mérito de Ortolani foi a divulgação do método de diagnóstico entre os pediatras.

Atualmente, há bastante conscientização sobre a necessidade do diagnóstico e tratamento precoces da DDQ e que o primeiro passo para isso deve ser feito na sala de parto e na maternidade. 


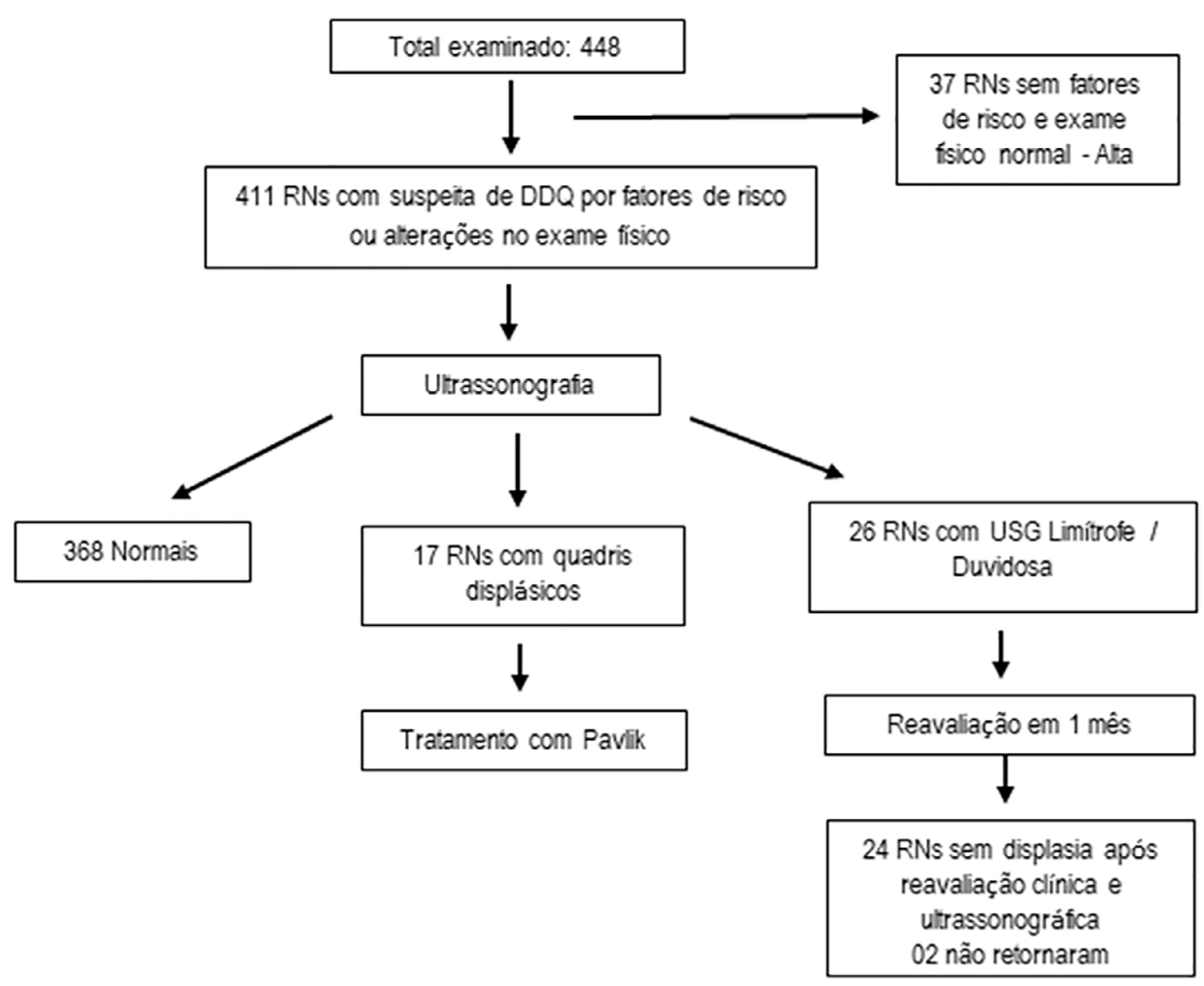

Figura 3. Caracterização da amostra e resultados.

Entretanto, menos conhecido é o fato de que o quadril deve continuar sendo examinado no seguimento de puericultura, até os seis meses de idade, para o diagnóstico dos casos tardios, mas com exame inicial normal. O objetivo da presente investigação foi avaliar o primeiro período do diagnóstico, ou seja, na fase de recém-nascido. Nossos resultados gerais mostram que o grupo de pediatras da maternidade em questão está bem esclarecido sobre a importância do diagnóstico. Outro ponto importante é fornecer ao pediatra um canal de encaminhamento dos casos para que a avaliação pelo ortopedista seja fácil. Neste aspecto também nossos dados mostram que há uma adequação desta sistemática, pois os recém-nascidos foram examinados na faixa etária adequada.
Porém, uma limitação metodológica é que não temos informação sobre os casos nascidos na maternidade, não suspeitos de instabilidade do quadril, e, portanto, não encaminhados, embora a condição pudesse existir (falso negativos). Publicações recentes alertam que qualquer profissional, ou mesmo o exame ultrassonográfico, não consegue diagnosticar todos os casos de instabilidade ${ }^{17}$, mesmo porque a afecção pode ter várias nuances e ser tão tênue no recém-nascido que é indetectável ao exame clínico, mas pode progredir com o crescimento. Por esta razão os pediatras devem ser conscientizados de que a pesquisa da displasia do quadril não termina no berçário, mas deve se prolongar ao longo da puericultura. 
Assim, a possibilidade de diagnóstico de casos de manifestação tardia fica aumentada. Também, esses mesmos profissionais devem estar cientes de que a displasia do quadril manifesta-se de maneira diferente, conforme a idade. Com efeito, o sinal de Ortolani deve ser pesquisado nas primeiras 48 horas de vida para ser mais sensível, depois desaparece, em torno dos dois meses, para ser substituído pela limitação da abdução do quadril (sinal de Hart), assimetria de pregas glúteas (sinal de Peter-Bade) e encurtamento (sinal de Galeazzi). Entretanto, nos graves casos de luxação teratogência esses sinais estão presentes já no recém-nascido. Quando a deambulação for iniciada, pode surgir a marcha por insuficiência do glúteo médio (Trendelenburg).

No Brasil, Souza et al. ${ }^{18}$ concluíram que o conhecimento da DDQ entre os profissionais da saúde que realizam a triagem no recém-nascido é falho, visto que $81,1 \%$ deles (estudantes, residentes, pediatras e ortopedistas) nunca fizeram um diagnóstico de DDQ. Neste sentido, o uso de manequins pode ser bastante útil para o treinamento de estudantes e pessoal da área da saúde, uma vez que a condição é pouco frequente e mesmo ortopedistas podem não ter experiência com nenhum caso.

Em nosso protocolo, são valorizados os sinais de risco e o exame clínico precoce, para que sirvam não somente como diagnóstico, mas como critério de indicação do US. Nossos resultados mostram que houve excesso de suspeita ou diagnóstico de instabilidade do quadril pelo pediatra. Embora sobrecarregue o serviço de saúde, este comportamento é preferível porque dá à criança uma segunda oportunidade de avaliação. Uma revisão da Cochrane comparou os custos e os benefícios do rastreamento de quadris de recémnascidos pela avaliação clínica e por meio do US.
Quando o US foi realizado de rotina em todos os recém-nascidos não houve queda da incidência de casos tardios, porém, houve aumento do número de re-exames e da taxa de tratamento desnecessários, além de sobrecarga do sistema de saúde ${ }^{19-21}$. Segundo Paton ${ }^{22}$, a realização da triagem neonatal clínica de todos os recém-nascidos apresenta uma sensibilidade de 66\%, uma especificidade de 99,8\%, um Valor Preditivo Positivo (VPP) de $28 \%$ e um Valor Preditivo Negativo (VPN) de 99,9\%. A realização do exame ultrassonográfico nos neonatos com quadris considerados em risco para DDQ aumenta a sensibilidade para $100 \%$ e a especificidade para $94,2 \%$, e tem VPP de $20,5 \%{ }^{22}$. Kyung et al. ${ }^{23}$ recomendaram que a US seja realizada no rastreio da DDQ para avaliar a morfologia e a estabilidade, em acréscimo ao exame físico do recém-nascido, visto que cerca de $7 \%$ dos quadris normais ao exame físico apresentavam displasia ao exame ultrassonográfico.

Outro aspecto que deve ser apreciado é em relação ao chamado "clique" ou "estalido" do quadril. Isto confunde muito os pediatras e ortopedistas, que podem interpretá-lo como sinal de Ortolani e, mesmo na literatura, o significado deste achado é controverso ${ }^{24}$. Kamath e Bramley ${ }^{24}$ examinaram ultrassonograficamente casos de estalido e verificaram que, em uma pequena porcentagem, estavam associados à displasias leves, que não requeriam tratamento. Recomendaram que os cliques persistentes por mais de seis semanas deveriam ser investigados pela US. De qualquer forma, é recomendável que o exame ultrassonográfico seja sempre associado ao exame clínico ${ }^{25,26}$. Como conclusão, há várias nuances para os cliques. Aqueles mais típicos e persistentes até três meses depois do primeiro exame devem ser submetidos à US. 
Em nossos casos, 8,5\% dos recém-nascidos tinham estalido no primeiro atendimento, entretanto, nenhum deles persistiu e não houve necessidade de prolongar o seguimento por essa razão.

Levando-se em conta que o quadril é uma articulação tão estável no adulto, é intrigante que apresente esse grau de instabilidade na criança pequena. Mais ainda, se considerarmos que o quadril tem origem embriológica em uma massa mesenquimal única, que se diferencia progressivamente no sentido de individualizar o fêmur do acetábulo, com a criação de uma fenda entre os núcleos mesenquimais, ou seja, a cabeça do fêmur forma-se originalmente dentro do acetábulo27. Isso leva a buscar algum fator que pudesse alterar essa relação após a formação da articulação.
Acredita-se que haja dois fatores importantes nos casos de DDQ: a displasia do acetábulo e a frouxidão capsulo-ligamentar ${ }^{27}$. Atualmente, o diagnóstico e tratamento são feitos com base nas alterações morfológicas do acetábulo, mas, provavelmente, essa alteração seja secundária ao posicionamento dinâmico inadequado da cabeça femoral antes do nascimento, em virtude de exagerada complacência da cápsula, em alguns indivíduos.

Em resumo, nossos resultados mostram que houve excesso de diagnóstico de instabilidade do quadril na avaliação do pediatra e, consequentemente, encaminhamento ao ortopedista. Embora possa causar sobrecarga ao serviço de saúde, esta atitude é adequada, pois permite ao paciente uma segunda avaliação em um ambiente mais especializado e com mais recursos tecnológicos.

\title{
A B S T R A C T
}

\begin{abstract}
Objective: to evaluate newborns with suspected hip instability, referred by pediatricians to a tertiary orthopedic service. Methods: newborns from a public university maternity hospital, with suspected instability or risk factors for hip dysplasia, were referred to the Department of Orthopedics and Anesthesiology, Ribeirão Preto/SP, where we evaluated them clinically and through ultrasound examinations of the hips. Once we found dysplasia, we initiated treatment, and in cases in which there was only hip immaturity and normal clinical examination, we performed clinical and ultrasound observation and review at two or three months of age. Results: we examined 448 newborns, with female predominance and average age at first evaluation of 27 days. The main cause of referral was pelvic presentation at delivery. In $8 \%$ there was a positive Ortolani sign and in 12.5\%,. At orthopedic examination, 405 (90.5\%) patients were normal, $8.5 \%$ had hip click and 1.1\% had positive Ortolani test. At ultrasound, 368 (89.5\%) had immaturity, $26(6.3 \%)$ had moderate dysplasia and in 17 (4.1\%) patients the hips were frankly dysplastic. All cases with positive Ortolani sign showed dysplasia at ultrasound. Conclusion: there was an excess diagnosis of hip instability in the pediatrician evaluation, which, however, allowed the patient a second assessment, in a more specialized environment and with more technological resources.
\end{abstract}

Keywords: Infant, Newborn. Hip. Hip Dislocation, Congenital. Joint Dislocations. Joint Instability.

\section{REFERÊNCIAS}

1. Pollet $\mathrm{V}$, Percy $\mathrm{V}$, Prior HJ. Relative risk and incidence for developmental dysplasia of the hip. J Pediatr. 2017;181:202-7.

2. $\quad$ Puech R. O problema da luxação congênita do quadril no Brasil. Tema oficial do II Congresso da Sociedade Brasileira de Ortopedia e Traumatologia, 1937. São Paulo: Empresa Gráfica da Revista dos Tribunais; 1937.

3. Lehmann HP, Hinton R, Morello P, Santoli J. Developmental dysplasia of the hip practice guideline: technical report. Committee on Quality Improvement, and Subcommittee on Developmental Dysplasia of the Hip. Pediatrics. 2000;105(4):e57.
4. Ortiz-Neira CL, Paolucci EO, Donnon T. A metaanalysis of common risk factors associated with the diagnosis of developmental dysplasia of the hip in newborns. Eur J Radiol. 2012;81(3):e344-51.

5. Patel $\mathrm{H} ;$ Canadian Task Force on Preventive Health Care. Preventive health care, 2001 update: screening and management of developmental dysplasia of the hip in newborns. CMAJ. 2001;164(12):1669-77.

6. Howorth B. The etiology of congenital dislocation of the hip. Clin Orthop. 1963;29(17):164-79.

7. Klisic PJ. Congenital dislocation of the hips-a misleading term: brief report. J Bone Joint Surg Br. 1989;71(1):136 
8. Graf R. The diagnosis of congenital hip-joint dislocation by the ultrasonic Combound treatment. Arch Ortop Trauma Surg. 1980;97(2):117-33.

9. Graf R. Fundamentals of sonographic diagnosis of infant hip dysplasia. J Pediatr Orthop. 1984;4(6):735-40.

10. Harcke HT, Clarke NM, Lee MS, Borns PF, MacEwen GD. Examination of the infant hip with real-time ultrasonography. J Ultrasound Med. 1984;3(3):131-7.

11. Graf R. Classification of hip joint dysplasia by means of sonography. Arch Orthop Trauma Surg. 1984;102(4):248-55.

12. Scapinelli $R$, Ortolani M. La displasia congenita delle anche nell'eta pediatrica. Societá Italiana di Ortopedia e Traumatologia. 1972;80-4.

13. Barlow TG. Early diagnosis and treatment of congenital dislocation of the hip. J Bone Joint Surg Br. 1962;44(2):292-301

14. Pavlik A. The functional method of treatment using a harness with stirrups as the primary method of conservative therapy for infants with congenital dislocation of the hip. Z Orthop Ihre Grenzgeb. 1957;89:341-52.

15. Le Vay D. History of orthopaedics: an account of the study and practice of orthopedics from the earliest times to the Modern Era. England: Parthenon publishing group limited; United Kingdom: Parthenon publishing group; 1990. Chapter 5 National Histories - Italy.

16. Le Vay D. History of orthopaedics: an account of the study and practice of orthopedics from the earliest times to the Modern Era. England: Parthenon publishing group; 1990. Chapter 4 - National Histories - France.

17. Kotlarsky P, Haber R, Bialik V, Eidelman $M$. Developmental dysplasia of the hip: what has changed in the last 20 years? World J Orthop. 2015;6(11):886-901.

18. Souza BGSE, de Melo TE, Resende TM, da Silva RCR, Cruz SA, de Oliveira VM. Developmental dysplasia of the hip: do the responsible for screening know what to do? Acta Ortop Bras. 2016;24(6):312-7.
19. Shorter D, Hong T, Osborn DA. Cochrane Review: Screening programmes for developmental dysplasia of the hip in newborn infants. Evid Based Child Health. 2013;8(1):11-54.

20. Rosendahl K, Markestad T, Lie RT. Ultrasound screening for developmental hip dysplasia in the neonate: the effect on treatment rate and prevalence of late cases. Pediatrics. 1994;94(1):47-52.

21. Bialik V, Bialik GM, Wiener F. Prevention of overtreatment of neonatal hip dysplasia by the use of ultrasonography. J Pediatr Orthop B. 1998;7(1):39-42.

22. Paton RW. Screening in Developmental Dysplasia of the Hip (DDH). Surgeon. 2017;15(5):290-6.

23. Kyung BS, Lee SH, Jeong WK, Park SY. Disparity between clinical and ultrasound examinations in neonatal hip screening. Clin Orthop Surg. 2016;8(2):203-9.

24. Kamath S, Bramley D. Is 'clicky hip' a risk factor in developmental dysplasia of the hip? Scott Med J. 2005;50(2):56-8.

25. McClure P, Podeszwa DA. Hip click in the neonate: should i be concerned? Pediatr Ann. 2016;45(4):e122-7.

26. Shaw BA, Segal LS; SECTION ON ORTHOPAEDICS. Evaluation and referral for developmental dysplasia of the hip in infants. Pediatrics. 2016;138(6):pii:e20163107.

27. Volpon JB. Desenvolvimento e anatomia do quadril. In: Pardini, Souza G, Laredo Filho J. Clinica Ortopédica. Rio de Janeiro: MEDSI; 2001. p. 11-20.

Recebido em: 28/06/2019

Aceito para publicação em: 17/09/2019

Conflito de interesse: nenhum.

Fonte de financiamento: nenhuma.

\section{Endereço para correspondência:}

José Batista Volpon

E-mail: hc.ortopedia@gmail.com

m.gutocruz@gmail.com

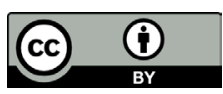

